\title{
Shear rate dependence and the effect of resistivity in magneto-rotationally unstable, stratified disks
}

\author{
U. Ziegler and G. Rüdiger
}

Astrophysikalisches Institut Potsdam, An der Sternwarte 16, 14482 Potsdam, Germany

Received 2 November 2000 / Accepted 3 September 2001

\begin{abstract}
We study the effect of resistivity and variation of shear rate, $q=-\mathrm{d} \log \Omega / \mathrm{d} R$, on the magnetorotational instability in stratified disks. Special attention is paid to dynamo $\alpha$-action and transport properties of the flow. The 3D magnetohydrodynamical simulations are performed in the local shearing-box approximation employing the code NIRVANA. Due to the chosen type of vertical boundary condition a mean azimuthal magnetic field is generated which is related to dynamo $\alpha$-action. The corresponding $\alpha$-parameter is negative (positive) in the northern (southern) disk hemisphere. This result is independent of $q$ ranging between 0.6 and 1.5 and independent of the amount of diffusivity. The magnitude of $\alpha$ typically declines with decreasing $q$. We suggest a natural limit in the vicinity of $q=0.6$ below which $\alpha$-action may be suppressed completely. Resistivity has a significant influence on the magnetic energy level of the final state. It is reduced for decreasing magnetic Reynolds number, $R e_{\mathrm{m}}$, as one might expect. However, the magneto-rotational instability is able to sustain an angular momentum transport of sufficient amount against diffusion even for the most diffusive case we considered, with $R e_{\mathrm{m}}=200$. The ShakuraSunyaev parameter in this case is roughly $1 / 3$ of that of the non-resistive model with the dominant contribution provided by the Reynolds stress rather than Maxwell stress. The dynamo $\alpha$-effect is found to vanish for strong dissipation.
\end{abstract}

Key words. magnetic fields - instabilities - MHD - turbulence

\section{Introduction}

A great step forward in our understanding of accretion disk inner workings has been the rebirth of the so-called magneto-rotational instability (MRI) by Balbus \& Hawley (1991). The MRI in its global form was known decades before (Velikhov 1959; Chandrasekhar 1960) but its fundamental meaning as a natural source of turbulence was not recognized at that time. Nowadays, numerical simulations showed that the MRI can explain a large amount of the anomalous viscosity in accretion disks (Hawley et al. 1995; Stone et al. 1996; Abramowicz et al. 1996; Brandenburg 1998). The long-standing theoretical puzzle of angular momentum transport in those objects thereby seems to be solved.

The question of a dynamo-excited magnetic field is as important as the understanding of the origin of outward angular momentum transport. Several numerical studies based upon different codes have demonstrated that the MRI is capable of amplifying a seed magnetic field whose field energy is susceptible to decay freely (Brandenburg et al. 1995, hereafter BNST; Hawley et al. 1996, hereafter HGB; Ziegler \& Rüdiger 2000, hereafter ZR). The presence of substantial (numerical) dissipation in those

Send offprint requests to: U. Ziegler,

e-mail: uziegler@aip.de simulations suggests that a fully self-consistent, hydromagnetic dynamo is operating. The models of BNST and ZR also yield the development of a mean magnetic field in the azimuthal direction which shows a cyclic behavior in time. This mean field is found to be correlated with the mean turbulent electromotive force (EMF), $\left\langle\boldsymbol{u}^{\prime} \times \boldsymbol{B}^{\prime}\right\rangle$, resulting from the interaction between the fluctuating velocity $\boldsymbol{u}^{\prime}$ and fluctuating magnetic field $\boldsymbol{B}^{\prime}$; a clear indication of the presence of a dynamo $\alpha$-effect.

The idea of generating large-scale magnetic fields through an $\alpha$-effect is a basic concept in the theory of turbulent dynamos. Loosely speaking, the quantity $\alpha$ encapsulates all the coherent effects of small-scale motions on magnetic field amplification. Mathematically,

$\left\langle\boldsymbol{u}^{\prime} \times \boldsymbol{B}^{\prime}\right\rangle=\alpha\langle\boldsymbol{B}\rangle+$ higher derivatives of $\langle\boldsymbol{B}\rangle$,

where $\langle\boldsymbol{B}\rangle$ denotes the mean magnetic field (Krause \& Rädler 1980). In the non-linear MRI simulations of ZR the resulting $\alpha$-effect turned out to be very noisy. Nevertheless, $\alpha$ could be assigned a definite sign; it is negative in the northern hemisphere of the disk and positive in the southern hemisphere i.e. $\alpha$ changes sign at the disk's midplane as might be expected from general principles. Kinematic theory predicts positive $\alpha$ in the northern hemisphere and negative $\alpha$ in the southern hemisphere and, thus, is in contradiction to the finding of $\mathrm{ZR}$ and 
BNST. Obviously, the simple kinematic picture fails to describe MRI-driven accretion disk dynamos.

The purpose of this paper is to examine the non-linear stage of the MRI in vertically stratified disks with a focus on the influence of magnetic diffusivity and of the strength of background shear. One major aspect concerns the derivation of the dynamo $\alpha$-coefficient, if applicable, based on description (1) under these different physical conditions. A series of both ideal and resistive MHD simulations is presented. With the inclusion of magnetic diffusion it is applied to account for situations where an ideal MHD is inadequate. This occurs in cold, dense plasma which is found, for instance, in the central regions of protoplanetary disks (Stone et al. 2000). All simulations enter far into the non-linear regime of the MRI and cover several tens of rotational periods. Our longest run has been extended up to several hundred orbits.

The format of the paper is as follows. In Sect. 2, the local ansatz, initial- and boundary conditions are discussed. Results of the simulations are presented in Sect. 3. Our conclusions are given in Sect. 4.

\section{The numerical model}

\subsection{Local approach and initial conditions}

As in most prior numerical work on the non-linear evolution of the MRI in accretion disks, the shearing-box approximation is used. This means that, instead of modeling the whole disk, a 3D rectangular box, located at a fiducial radius $R_{0}$ and with linear extent much less than $R_{0}$, is considered. The equations of magnetohydrodynamics are solved in a corotating Cartesian frame of reference with its origin at the center of the box. Locally, the unit vector $\hat{\boldsymbol{x}}$ is oriented in the radial direction, $\hat{\boldsymbol{y}}$ in the azimuthal direction and $\hat{z}$ parallel to the disk rotation axis. The magnetohydrodynamical equations in this local frame are the following:

$$
\begin{aligned}
& \frac{\partial \rho}{\partial t}+\nabla \cdot(\rho \boldsymbol{u})=0 \\
& \frac{\partial(\rho \boldsymbol{u})}{\partial t}+\nabla \cdot(\rho \boldsymbol{u u})=-\nabla p+\frac{1}{\mu}(\nabla \times \boldsymbol{B}) \times \boldsymbol{B} \\
& -2 \rho \Omega \hat{\boldsymbol{z}} \times \boldsymbol{u}+2 \rho q \Omega^{2} x \hat{\boldsymbol{x}}-\rho g \hat{\boldsymbol{z}}, \\
& \frac{\partial e}{\partial t}+\nabla \cdot(e \boldsymbol{u})=-p \nabla \cdot \boldsymbol{u}+\frac{\eta}{\mu}|\nabla \times \boldsymbol{B}|^{2}, \\
& \frac{\partial \boldsymbol{B}}{\partial t}=\nabla \times(\boldsymbol{u} \times \boldsymbol{B}-\eta \nabla \times \boldsymbol{B}) .
\end{aligned}
$$

$\rho$ is the gas density, $p$ the gas pressure which is related to the thermal energy density $e$ through the equation of state $p=(\gamma-1) e$ with $\gamma=5 / 3, \boldsymbol{u}$ denotes the flow velocity and $\boldsymbol{B}$ is the magnetic field. The parameter $q=-\mathrm{d} \log \Omega / \mathrm{d} \log R$ is a measure of the local shear rate derived from the disk rotation law $\Omega(R)$. The term $-\rho g \hat{z}$ represents the vertical gravitational force. For a thin Keplerian disk $g=\Omega^{2} z$ and $q=1.5$. The $+2 \rho q \Omega^{2} x \hat{\boldsymbol{x}}$ term incorporates tidal forces and results from an expansion of the effective (gravitational+centrifugal) potential in the corotating reference frame for $x \ll R$. Ultimately, $\mu$ represents the magnetic permeability which is set to its vacuum value $\mu=\mu_{0}=4 \pi \times 10^{-7} \mathrm{Vs} / \mathrm{Am}$, and $\eta$ denotes the magnetic diffusivity. Instead of $\eta$, the magnetic Reynolds number $R e_{\mathrm{m}}$ is introduced as a control parameter of the simulations. Our definition of $R e_{\mathrm{m}}$ is based on the characteristic velocity of the initial shear flow, $q \Omega L_{x}$, where $L_{x}$ is the box dimension in the $x$-direction, and on the disk scale height $H_{0}$ (see below):

$R e_{\mathrm{m}}=\frac{q \Omega L_{x} H_{0}}{\eta}$

Alternatively, one could use the sound speed, which is initially constant, as the characteristic velocity in definition (6). The choice of parameters below the magnetic Reynolds number in this case is a factor of $q \sqrt{2} \approx 2$ smaller for $q=1.5$.

The equations are solved with the 3D AMR-MHD code NIRVANA (Ziegler 1998; Ziegler 1999) using a uniform grid of dimension $32 \times 64 \times 64$. The AMR feature of the code is not used in this study. The grid spans the spatial (dimensionless) domain $(x, y, z) \in[-1 / 2,1 / 2] \times[0,2 \pi] \times$ $[-2,2]$. The zone spacings behave like $\delta x: \delta y: \delta z=1: \pi: 2$. Because of the moderate numerical resolution, dissipative effects due to the truncation error of the code are surely of some importance. As the present study does not include a physical viscous dissipation which dominates numerical dissipation, the effect of viscosity cannot be described correctly within our approach. To obtain a rough estimate of the influence of viscous forces, explicit artificial viscosity due to von Neumann-Richtmyer is added which may or may not be turned on. Generally, artificial viscosity plays a dual role. It first dissipates high-frequency numerical noise and, second, it smears out shock fronts if the flow becomes supersonic. Here, the damping effect on the turbulent motion is the more important property. The von Neumann-Richtmyer viscosity enters the equation of motion and energy balance equation as an anisotropic pressure (not shown in the equations) given by

$q_{i}= \begin{cases}l \rho\left(\frac{\partial u_{i}}{\partial x_{i}}\right)^{2}\left(\delta x_{i}\right)^{2} & \text { if }\left(\frac{\partial u_{i}}{\partial x_{i}}\right)<0, \\ 0 & \text { otherwise. }\end{cases}$

The "viscosity parameter" $l$ is related to the number of grid zones over which a shock is spread and is typically of the order of unity.

The initial disk stratification is assumed to be isothermal with the Gaussian density profile

$\rho(z)=\rho_{0} \exp \left\{-z^{2} / H_{0}^{2}\right\}$

where $\rho_{0}$ is the density in the central plane of the disk and $H_{0}$ is its typical scale height. A hydrostatic atmosphere is achieved by the choice $g=2 c_{\mathrm{S}}^{2} / H_{0}^{2} z$, where $c_{\mathrm{S}}$ denotes the initial (constant) sound speed. Similar to previous MHD simulations (BNST, ZR), we adopt dimensionless quantities by putting $\rho_{0}=H_{0}=G M=1$ ( $G$ : gravitation constant, $M$ : mass of central object). In all simulations $\Omega=10^{-3}$ and $c_{\mathrm{S}}^{2}=5 \times 10^{-7}\left(=p_{0}\right.$, the pressure at 
$z=0)$. The initial configuration is completed by specifying the velocity of the background shear flow which is given by $\boldsymbol{u}_{0}=\left(u_{x 0}, u_{y 0}, u_{z 0}\right)=(0,-q \Omega x, 0)$.

The disk atmosphere is threaded by a magnetic field which is purely vertical and varies sinusoidally in the $x$-direction:

$\boldsymbol{B}=B_{0} \sin (2 \pi x) \hat{\boldsymbol{z}}$.

In dimensionless units the field amplitude is chosen has $B_{0}=1.121 \times 10^{-7}$ which corresponds to a plasma- $\beta$ of $\beta=2 \mu p_{0} / B_{0}^{2}=100$ at the disk midplane. For a constant $\beta$ the fastest growing unstable wavelength of the MRI from Balbus \& Hawley (1991) is $\lambda_{\mathrm{f}} \approx 9.18 c_{\mathrm{S}} / \Omega \sqrt{\beta} \approx 11 \delta z$. The mode $\lambda_{\mathrm{f}}$ is therefore well-resolved on the numerical grid. Here, because $\beta$ is a function of height, $\lambda_{\mathrm{f}}$ increases to $\approx 80 \delta z$ at $z \pm 2$ which exceeds the vertical extent of the computational domain.

\subsection{Boundary conditions}

Shear-periodic boundary conditions are adopted in the $x$ direction to account for the large-scale shear across the box (for more detail see Hawley et al. 1995). Thereby, at $x= \pm 1 / 2$ the basic variables obey the relations

$f( \pm 1 / 2, y, z)=f(\mp 1 / 2, y \pm q \Omega t, z)$

for $f=\rho, e, u_{x}, u_{z}, B_{x}, B_{y}, B_{z}$ and

$u_{y}( \pm 1 / 2, y, z)=u_{y}(\mp 1 / 2, y \pm q \Omega t, z) \mp q \Omega$.

Straightforward periodic boundary conditions are assumed in $y$-direction. In the vertical direction, we adopt

$$
\frac{\partial \rho}{\partial z}=\frac{\partial e}{\partial z}=\frac{\partial u_{x}}{\partial z}=\frac{\partial u_{y}}{\partial z}=u_{z}=B_{x}=B_{y}=\frac{\partial B_{z}}{\partial z}=0 .
$$

The condition on the velocity ensures the absence of stress in the flow. Furthermore, the magnetic field is forced to be perpendicular at the lower and upper $z$-boundary but otherwise the magnetic field lines can move freely across the surface.

This special choice of vertical boundary condition has crucial consequences for the evolution of the mean magnetic field $\langle\boldsymbol{B}\rangle$. If this mean is interpreted as the spatial average over the computational domain, one obtains from the induction equation after some algebraic manipulations

$$
\begin{aligned}
& \partial_{\mathrm{t}}\left\langle B_{x}\right\rangle=\left\langle\partial_{z}\left(u_{x} B_{z}\right)\right\rangle, \\
& \partial_{\mathrm{t}}\left\langle B_{y}\right\rangle=\left\langle\partial_{z}\left(u_{y} B_{z}\right)\right\rangle-\left.\frac{q \Omega}{4 \pi} \iint \mathrm{d} y \mathrm{~d} z B_{x}\right|_{x=-\frac{1}{2}}, \\
& \partial_{\mathrm{t}}\left\langle B_{z}\right\rangle=0 .
\end{aligned}
$$

Hence, whereas the mean vertical component remains unchanged for all times, the two horizontal components are not but are allowed to vary with time even if the mean magnetic field vanishes initially. This will indeed happen for our choice of magnetic field configuration.

The existence of a dynamo $\alpha$-effect is tied to the presence of a mean magnetic field. The generation of a mean magnetic field, in turn, is favored by our choice of boundary conditions. Because it is hardly possible to separate the various physical effects from effects caused by the numerical boundary conditions, one might ask to what extent the results are then influenced particularly by our choice of vertical boundary conditions. Although mathematically well posed, the unkind point of (12) is that horizontal magnetic field structures in the vicinity of $z= \pm 2$ give rise to tension forces which may produce strong surface currents; a consequence of the fact that the magnetic field is forced to be perpendicular there. Those currents are somewhat artificial and are not necessarily real in accretion disks which constitute open systems. To avoid such boundary problems an ideal outflow condition, which exactly treats wave propagation across a surface, would be a better choice. However, such an ansatz is hard to implement and we dispense with it for the moment.

\section{Results}

We have performed a number of simulations which are specified by the three parameters $q$ (shear rate), $l$ (strength of artificial viscosity) and $R e_{\mathrm{m}}$ (magnetic Reynolds number). Note that in the non-resistive models $\left(R e_{\mathrm{m}}=\infty\right)$ numerical dissipation is still present, hence, the effective magnetic Reynolds number $R e_{\mathrm{m}, \mathrm{eff}}$ is finite. The complete sample of simulations is listed in Table 1. In addition to model parameters, Table 1 contains the number of orbits each simulation has been advanced in time and several fundamental quantities which are referred to later in the text. Mean quantities of the solution are obtained by averaging variables over the box volume or parts of it. The following convention applies for the rest of the paper: mean values taken over the total box volume are enclosed by brackets, $\langle$.$\rangle . The symbols \langle.\rangle^{\mathrm{N}}$ and $\langle.\rangle^{\mathrm{S}}$, on the other hand, represent mean values obtained by averaging over the northern disk hemisphere (superscript $\mathrm{N}$, $z>0$ ) respective southern disk hemisphere (superscript $\mathrm{S}$, $z<0)$. Time averages are denoted by overbars.

\subsection{Non-resistive Kepler disks}

This section is focused on the models labeled S0 (respectively, S0l) and S1 in Table 1. Model S0l is a continuation of run S0 extending its simulation time up to 288 orbits. This run has been performed in order to examine the behavior of the (in a statistical sense) steady state solution which can be seen to develop after a few rotational orbits over a longer timespan. A Keplerian background shear flow $(q=1.5)$ and an ideally conducting plasma, $R e_{\mathrm{m}}=\infty$, is assumed. These runs primarily serve as a reference for the models with different $q$ and different $R e_{\mathrm{m}}$ which are discussed later. S1 includes artificial viscosity adopting a viscosity parameter of $l=2$ whereas artificial viscosity is turned off in S0 $(l=0)$.

Figure 1 illustrates the time history of two important box-averaged quantities. The magnetic energy density and the $x y$-component of the Maxwell stress tensor - the 
Table 1. The sample of simulations.

\begin{tabular}{llllllll}
\hline \hline model & orbits & $q$ & $R e_{\mathrm{m}}$ & $l$ & $M R S R$ & $\alpha_{\mathrm{SS}}$ & $\left(\alpha^{* \mathrm{~N}}, \alpha^{* \mathrm{~S}}\right)$ \\
\hline $\mathrm{S} 0$ & 85 & 1.5 & $\infty$ & 0 & 3.5 & $9 \times 10^{-3}$ & $(-0.0025,0.0038)$ \\
$\mathrm{S} 01^{a}$ & 288 & 1.5 & $\infty$ & 0 & 3.8 & $10^{-2}$ & $(-0.0026,0.0038)$ \\
$\mathrm{S} 1$ & 85 & 1.5 & $\infty$ & 2.0 & 4.1 & $1.1 \times 10^{-2}$ & $(-0.0017,0.0024)$ \\
$\mathrm{S} 2$ & 84 & 1.0 & $\infty$ & 2.0 & 7.6 & $6.3 \times 10^{-3}$ & $(-0.0014,0.0020)$ \\
$\mathrm{S} 3$ & 30 & 0.7 & $\infty$ & 2.0 & 17.8 & $3.7 \times 10^{-3}$ & $(-0.0004,0.0008)$ \\
$\mathrm{S} 4$ & 27 & 0.6 & $\infty$ & 2.0 & 23.7 & $2.9 \times 10^{-3}$ & $(\approx 0, \approx 0)$ \\
$\mathrm{S} 5$ & 92 & 1.5 & 200 & 1.4 & 0.07 & $3.1 \times 10^{-3}$ & $(\approx 0, \approx 0)$ \\
$\mathrm{S} 6$ & 84 & 1.5 & 2000 & 1.4 & 0.78 & $3.7 \times 10^{-3}$ & $(-0.0030,0.0015)$ \\
$\mathrm{S} 7^{b}$ & 87 & 1.5 & 12000 & 1.4 & 1.03 & $3.3 \times 10^{-3}$ & $(-0.0017,0.0020)$ \\
$\mathrm{S} 7 \mathrm{P}^{b, c}$ & 96 & 1.5 & 12000 & 1.4 & 1.44 & $4.9 \times 10^{-3}$ & $(-0.0042,0.0041)$ \\
$\mathrm{H} 1^{b, c, d}$ & 72 & 1.5 & 12000 & 1.4 & 1.7 & $3.0 \times 10^{-2}$ & - \\
$\mathrm{H} 2^{b, c, d}$ & 91 & 1.5 & $\infty$ & 1.4 & 3.5 & $4.8 \times 10^{-2}$ & - \\
\hline & & & & & & & \\
\hline
\end{tabular}

a Time averages are restricted to $t<250$;

${ }^{b}$ Initial plasma beta $\beta=400$;

${ }^{c}$ Periodic boundary conditions in $z$-direction;

${ }^{d}$ Non-stratified box.
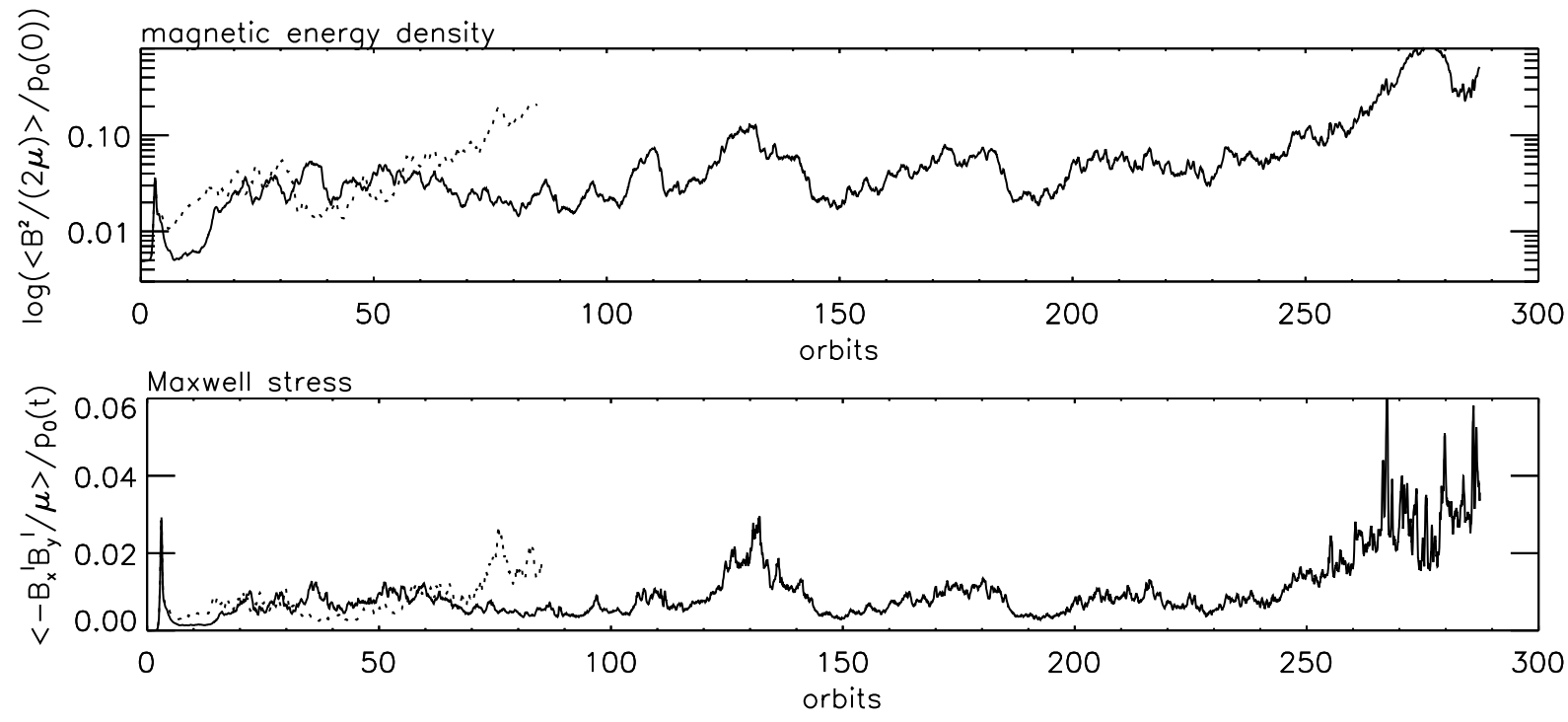

Fig. 1. Time history of volume-averaged (normalized) quantities. The logarithm of magnetic energy (top) and Maxwell stress (bottom) for the models S0l (solid line) and S1 (dotted line) are shown.

most relevant component for angular momentum transport - are shown. Note the scalings of these quantities: the magnetic energy density is normalized to $p_{0}$, whereas the Maxwell stress is scaled to the time-dependent, horizontally-averaged pressure at $z=0$ denoted by $p_{0}(t)$. The initially amplified magnetic field is maintained up to the end of the simulation. 288 orbits in S0l appears to be a rather long time; probably much longer than the resistive decay time due to pure numerical truncation in the NIRVANA code. Although we did not calibrate NIRVANA to prove this directly and to estimate corresponding decay rates, such experiments were performed by HGB for the ZEUS code (Stone \& Norman 1992a; Stone \& Norman 1992b). HGB hereby verified the maintenance of a magnetic field over a time period considerably longer than the effective decay time scale. As the numerics in NIRVANA and ZEUS is basically the same, it is plausible to assume that both codes have similar numerical dissipation and, therefore, yield comparable decay rates. Based on this argument one can say that the minimum requirement for a dynamo, that is maintaining a magnetic field in the presence of dissipation, is fulfilled. Our long-term run in particular, hence, supports the picture of a selfconsistent, dynamic accretion disk dynamo also suggested in other work (e.g. Stone et al. 1996; Brandenburg et al. 1996; Hawley \& Stone 1998). 


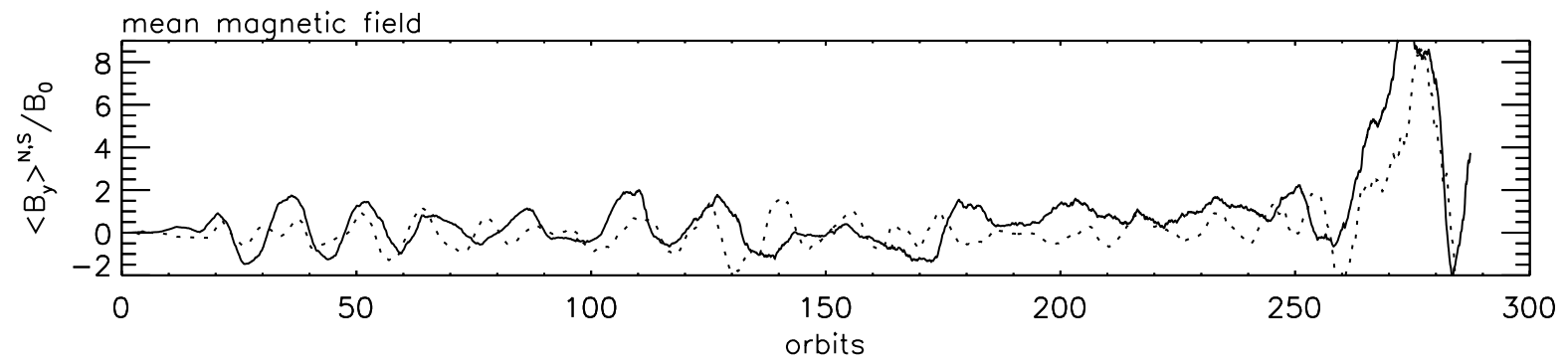

Fig. 2. Model S0l: time evolution of the mean azimuthal magnetic field component. The average is taken over both the northern disk hemisphere (solid line) and southern disk hemisphere (dotted line).

The role of artificial viscosity is that of an additional dissipation mechanism for turbulent motions. Many dynamo simulations attest to the importance of the magnetic Prandtl number $P r_{\mathrm{m}}$ defined as the ratio of kinematic viscosity to magnetic diffusivity. Since artificial viscosity adds to numerical viscosity, one can argue that the effective magnetic Prandtl number, $P r_{\mathrm{m}, \mathrm{eff}}$, is larger in $\mathrm{S} 1$ than in S0 (no artificial viscosity). From that one might expect a higher energy level for S1 consistent with a larger $P r_{\mathrm{m}, \text { eff }}$ (Pouquet \& Patterson 1978). This may explain the observed increase in the magnetic energy in model S1 for $t \gtrsim 60$ not present in S0.

We furthermore reproduce the known result that angular momentum transport is mediated mainly by Maxwell stress rather than Reynolds stress and that transport is directed outward on statistical average. To quantify this effect we define a Maxwell-to-Reynolds stress ratio:

$M R S R=\frac{\overline{\left\langle-B_{x}^{\prime} B_{y}^{\prime} / \mu\right\rangle}}{\overline{\left\langle\rho u_{x}^{\prime} u_{y}^{\prime}\right\rangle}}$.

If not otherwise stated, the time average is taken over the whole simulation time. We obtain a value of $M R S R=3.5$ (4.1) for S0 (S1). The total rate of angular momentum transport can be characterized by the Shakura-Sunyaev parameter $\alpha_{\text {SS }}$ (Shakura \& Sunyaev 1973):

$\alpha_{\mathrm{SS}}=\overline{\left\langle\frac{\rho u_{x}^{\prime} u_{y}^{\prime}-B_{x}^{\prime} B_{y}^{\prime} / \mu}{p_{0}(t)}\right\rangle}$.

The scaling of the total stress by gas pressure provides a route for comparison with the traditional $\alpha$ parameterization in accretion disk theory. It should be recalled, however, that the total stress and, thus, angular momentum transport, is more likely correlated with the magnetic pressure rather than gas pressure, as pointed out by HGB. We find values of $\alpha_{\mathrm{SS}}=9 \times 10^{-3}\left(10^{-2}\right)$ for S0 (S0l) and $\alpha_{\mathrm{SS}}=1.1 \times 10^{-2}$ for S1 which are consistent with previous work. The slightly higher values of $M R S R$ and $\alpha_{\mathrm{SS}}$ in the run including artificial viscosity again might be a consequence of the larger effective magnetic Prandtl number.

The vertical boundary condition permits the development of a mean horizontal magnetic field which is indeed generated. The plots in Fig. 2 illustrate the timedependence of $\left\langle B_{y}\right\rangle^{\mathrm{N}}$ and $\left\langle B_{y}\right\rangle^{\mathrm{S}}$. The radial component $\left\langle B_{x}\right\rangle$ is small compared to $\left\langle B_{y}\right\rangle$ and for the vertical component $\left\langle B_{z}\right\rangle=0$ as it must according to (15). The azimuthal mean field turns out quasi-periodic with sign reversals every $\approx 8$ orbits. The amplitude of the oscillation is of the order of $B_{0} \cdot\left\langle B_{y}\right\rangle^{\mathrm{N}}$ and $\left\langle B_{y}\right\rangle^{\mathrm{S}}$ are out of phase. In fact, there is no well-defined phase relation between these mean fields and the phase can be observed to change with time. For instance, $\left\langle B_{y}\right\rangle^{\mathrm{N}}$ and $\left\langle B_{y}\right\rangle^{\mathrm{S}}$ has the same orientation at $t \approx 50$ but opposite orientation at $t \approx 180$. Note that, although not explicitly visualized, artificial viscosity has only little influence on the cycle and amplitude of $\left\langle B_{y}\right\rangle$. BNST, using the same boundary conditions as we did, also found a cyclic behavior for $\left\langle B_{y}\right\rangle$ but with a larger period. As we will demonstrate in Sect. 3.3 the detailed time evolution of $\left\langle B_{y}\right\rangle$ is significantly affected by the amount of magnetic diffusion present.

The existence of $\left\langle B_{y}\right\rangle$ directs attention to the question of dynamo $\alpha$-action in the disk i.e. whether there exists a correlation between the mean field and the mean turbulent EMF. This is checked by evaluating relation (1) for a large ensemble of time snapshots - separately for the northern and southern disk hemisphere. One obtains for the $y$-component of the EMF:

$\left\langle E_{y}\right\rangle^{\mathrm{N}}=\left\langle u_{z}^{\prime} B_{x}^{\prime}-u_{x}^{\prime} B_{z}^{\prime}\right\rangle^{\mathrm{N}}=\alpha_{y y}^{\mathrm{N}}\left\langle B_{y}\right\rangle^{\mathrm{N}}$,
$\left\langle E_{y}\right\rangle^{\mathrm{S}}=\left\langle u_{z}^{\prime} B_{x}^{\prime}-u_{x}^{\prime} B_{z}^{\prime}\right\rangle^{\mathrm{S}}=\alpha_{y y}^{\mathrm{S}}\left\langle B_{y}\right\rangle^{\mathrm{S}}$.

$\alpha_{y y}^{\mathrm{N}}\left(\alpha_{y y}^{\mathrm{S}}\right)$ denotes the azimuthal $\alpha$-effect in the northern (southern) disk hemisphere. Note that, because $\left\langle B_{x}\right\rangle \ll$ $\left\langle B_{y}\right\rangle$ and $\left\langle B_{z}\right\rangle=0$, unimportant terms in (1) were dropped. In the following, $\alpha_{y y}^{\mathrm{N}}\left(\alpha_{y y}^{\mathrm{S}}\right)$ will be replaced by the shorter notation $\alpha^{\mathrm{N}}\left(\alpha^{\mathrm{S}}\right)$. The result of applying Eqs. (18) and (19) is presented in Fig. 3. Clearly, although highly noisy, an $\alpha$-effect is present. $\alpha$ changes sign with respect to the diskplane and is negative (positive) in the northern (southern) disk hemisphere. A linear fit through the sample yields for model S0

$\left(\begin{array}{l}\alpha^{* \mathrm{~N}} \\ \alpha^{* \mathrm{~S}}\end{array}\right)=\left(\begin{array}{c}-0.0025 \\ 0.0038\end{array}\right)$,

where $\alpha^{* \mathrm{~N}}\left(\alpha^{* \mathrm{~S}}\right)$ denotes the normalized $\alpha$-parameter measured in units of the initial sound speed $c_{\mathrm{S}}$. The values for S1 are approximately 30\% lower than those of S0 (see Table 1). Artificial viscosity in S1 likely reduces the EMF by dissipation of the fluctuating velocity $\boldsymbol{u}^{\prime}$ which, in turn, leads to a weaker $\alpha$-effect as observed. 

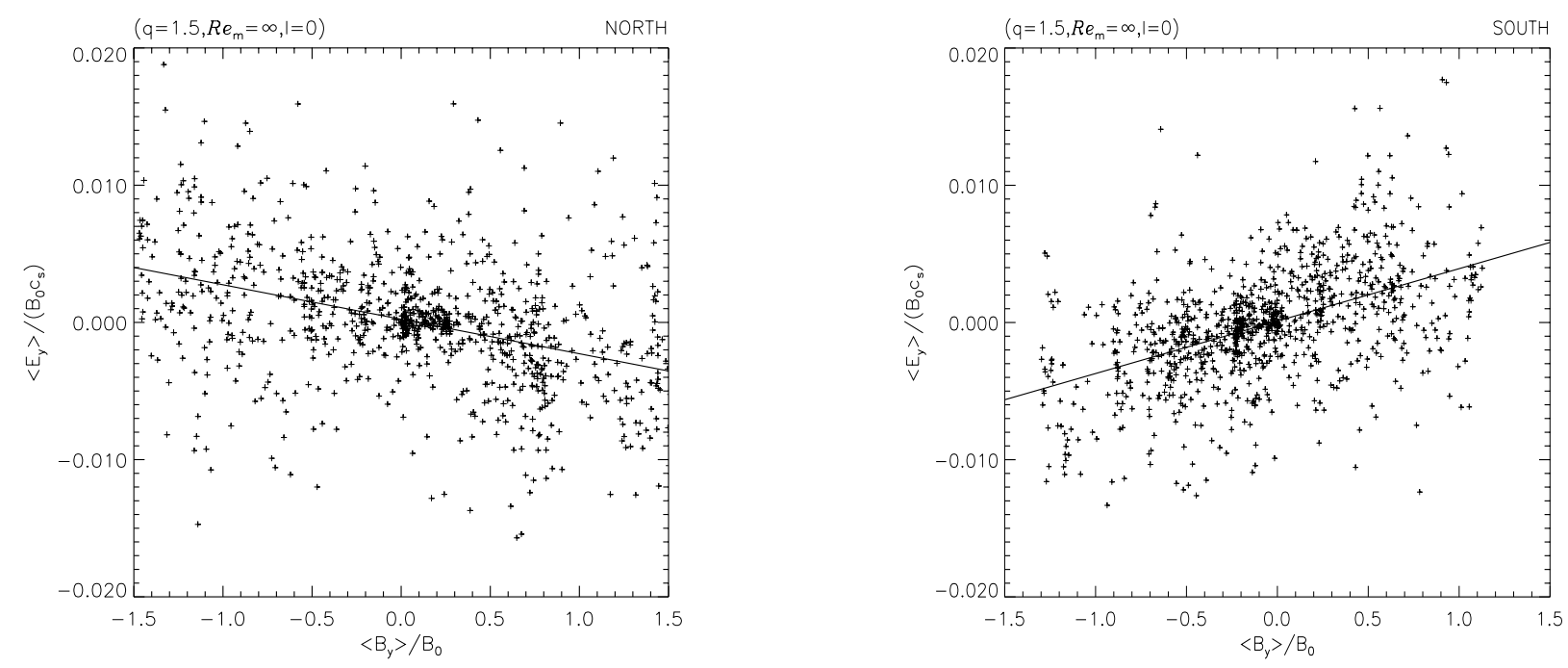

Fig. 3. $\alpha$-effect for model S0: correlation between the EMF and mean azimuthal field in the northern (left panel) and southern (right panel) disk hemisphere.

An interesting behavior is seen at late phases in the evolution of S0l. It manifests itself through a significant increase in the magnetic energy and strong fluctuations in the Maxwell stress beginning at $t \gtrsim 250$. At the same time, $\left\langle B_{y}\right\rangle$ grows by almost one order of magnitude, reaches a peak value of $\approx 8 B_{0}$ at $t \approx 277$, and then again declines within a timespan of $\approx 10$ orbits. A more detailed inventory of the data yields that at this stage the flow is surprisingly ordered. Motions in the $x z$-plane are predominantly radial ( $x$-direction). The ratio of turbulent kinetic energies in the $x$ - and $z$-direction is clearly in favor of the radial component,

$\left\langle\rho u_{x}^{2}\right\rangle /\left\langle\rho u_{z}^{2}\right\rangle \approx 8.7$

at $t \approx 277$. The flow structure is reminiscent of a $(y$ independent) 2D sandwich-like configuration where radial velocity streams are separated by more quiet regions, therefore, reminding us of the so-called channel solution (Goodman \& Xu 1994). These radial motions lead to strong magnetic field amplification and, in turn, to the observed increase in magnetic energy and Maxwell stress. Unfortunately, the time-step at this violent phase becomes too small so that the evolution cannot be followed further in a reasonable time. The final fate of the disk is therefore a matter of speculation. If the ordered flow persists, a hasty redistribution of matter would result which has an influence on the disk as a whole and which may, in the worst case, lead to its complete disruption (Stone \& Norman 1994). The ultimate proof of such a scenario, of course, lies beyond the possibilities of local simulations. It can be usefully addressed only by global simulations which are not of concern here.

\subsection{Dependence on the shear rate}

Having reviewed some fundamental properties of the MRI for Keplerian disks we now derive such properties for disks having a lower shear rate. It seems plausible to assume that the magnitude of the viscosity parameter $\alpha_{\mathrm{SS}}$ depends on the strength of the background shear because the growth rate of the MRI is proportional to $q$ (Balbus \& Hawley 1992). It can therefore be expected that $\alpha_{\mathrm{SS}}$ declines with decreasing $q$. Table 1 lists the resulting values of $\alpha_{\mathrm{SS}}$ for the cases $q=1.5,1.0,0.7,0.6$ corresponding to the runs labeled by S1, S2, S3, S4. In this series of runs $R e_{\mathrm{m}}=\infty$ and $l=2$. Indeed, the simulations confirm that $\alpha_{\mathrm{SS}}$ gradually decreases when $q$ changes from 1.5 to 0.6. In comparison to a Keplerian disk $\alpha_{\mathrm{SS}}$ is smaller by a factor of $\approx 2$ for a background rotational velocity profile with $q=0.6$. Along with the decrease in $\alpha_{\mathrm{SS}}$ there is an increase of the quantity $M R S R$. For $q=0.6$ we obtain a value of $M R S R \approx 24$ which is 6 times larger than in the Keplerian case. Hence, angular momentum transport is increasingly dominated by Maxwell stress rather than by Reynolds stress if the strength of the shear is reduced. Our results suggest the existence of a relation $\alpha_{\mathrm{SS}} \propto q$ in contrast to the finding of Abramowicz et al. (1996) obtaining a steeper profile.

The dependence of the dynamo $\alpha$-parameter on the shear rate is illustrated in Fig. 4. The cases $q=1.5,1.0,0.6$ are shown. The result for $q=0.7$ is not presented and lies somewhere in between the cases $q=1.0$ and $q=$ 0.6. There is an apparent trend to a smaller $\alpha$-effect as the shear rate declines. Most remarkably, $\left(\alpha^{* \mathrm{~N}}, \alpha^{* \mathrm{~S}}\right) \approx$ $(0,0)$ for $q=0.6$ while the electromotive force tends to zero. Note that the scatter of snapshot points typically becomes reduced for lower $q$. This is an indication of an increasingly diminishing time variability in the $\alpha$-effect and means that the corresponding $\alpha$-parameter becomes more clearly defined.

Rüdiger \& Pipin (2001) in a magnetically-driven turbulence model propose a dependence

$\alpha \propto\left(\frac{3}{4}-q\right)$

for the azimuthal dynamo $\alpha$-parameter on the shear rate including the effects of magnetic buoyancy and differential 

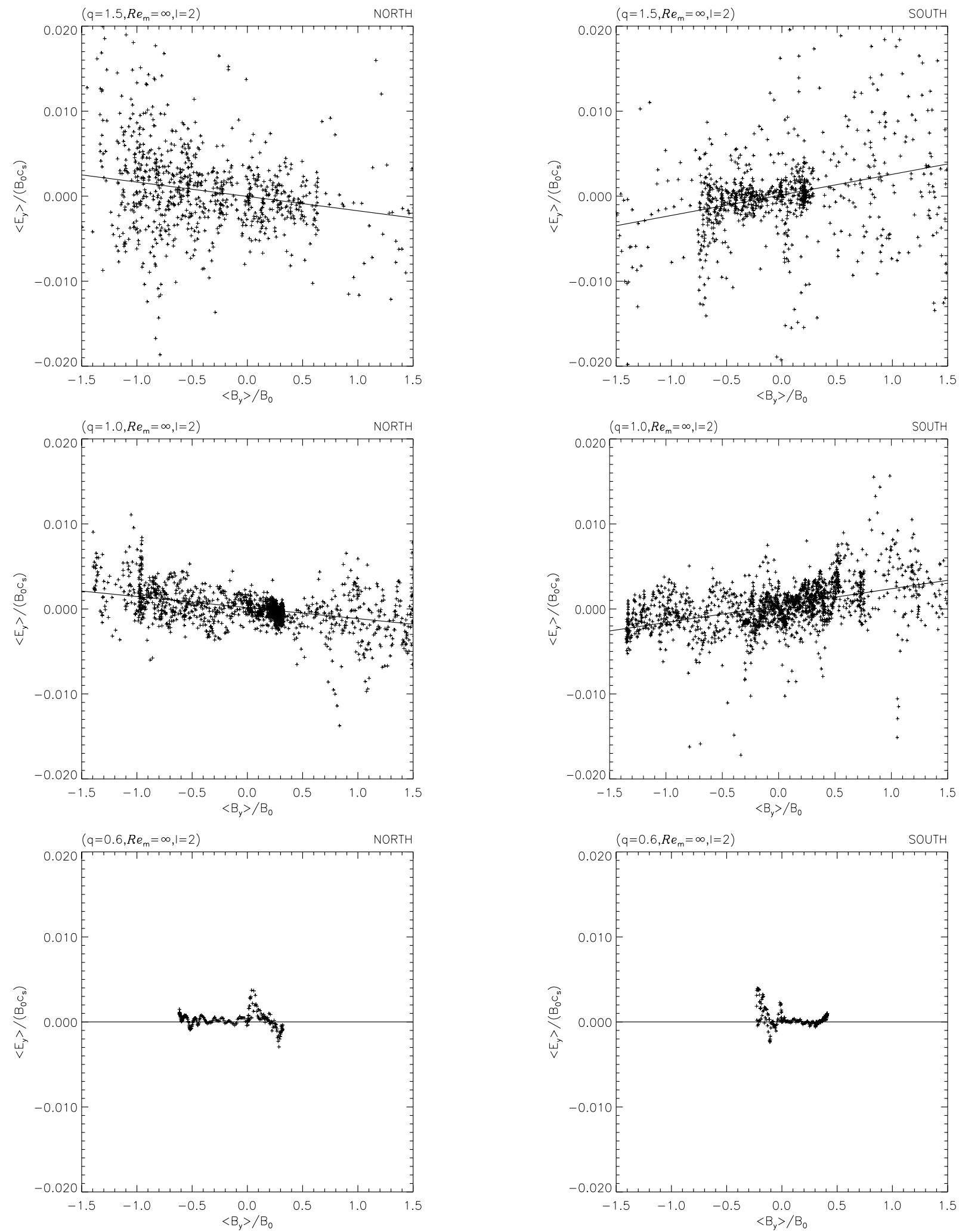

Fig. 4. $\alpha$-effect: correlation between the mean EMF and mean azimuthal field in the northern (left panel) and southern (right panel) disk hemisphere for the models S1 (top), S2 (middle) and S4 (bottom).

rotation. According to this analytical ansatz, $\alpha$ vanishes for $q=0.75$ which is due to the cancelation of the contributions resulting separately from the rotation and from the shear. On the other hand, it has been demonstrated that this cancelation effect does not influence $\alpha_{\mathrm{SS}}$. Our numerical simulations seem to support this idea. Here we find a non-vanishing $\alpha_{\mathrm{SS}}$ of significant amount but a vanishing dynamo $\alpha$-effect for $q=0.6$. This remarkable result likely means that the MRI is not able to drive largescale dynamos in disks that possess only a weak shear 
component. It does, however, not exclude dynamo action in general. Indeed, even in the case $q=0.6$ with a vanishing $\alpha$-parameter we observe dynamo activity in the sense of a growth in the magnetic field energy. This small-scale dynamo process implies a high enough Maxwell stress to allow transport of angular momentum at a rate $1 / 3$ of that of a Keplerian disk (see Table 1).

\subsection{The effect of magnetic diffusion}

In the past, MRI simulations with finite (physical) resistivity have been carried out by HGB and Fleming et al. (2000) (hereafter FSH). In contrast to the present simulations, neither HGB nor FSH include disk stratification. Instead, their models start with uniform density and pressure. Another important difference between their and our work is the choice of the vertical boundary condition that is periodic in HGB and FSH. It means that no net magnetic field can be generated in their simulations and, hence, no conclusions can be drawn about the existence of dynamo $\alpha$-action.

Here we present the results of the runs S5, S6, S7 characterized by magnetic Reynolds numbers $R e_{\mathrm{m}}=$ 200,2000, 12 000. A Keplerian background shear flow ( $q=$ $1.5)$ is assumed in all resistive models. Note that in S7 $\beta=400$ whereas $\beta=100$ in S5 and S6. We investigate the influence of the vertical boundary condition (12) by a comparison of models S7 and S7P. The latter uses periodic vertical boundary conditions but otherwise is identical to S7. Furthermore, to estimate the role of disk stratification two additional runs $(\mathrm{H} 1, \mathrm{H} 2)$ are performed having constant density and pressure throughout the box volume.

We want to start by giving some qualitative arguments on how the non-linear stage of the MRI could be affected by magnetic diffusion. The resistive damping rate of a given unstable mode of the MRI with wavelength $\lambda$ is typically of the order of $4 \pi^{2} \eta / \lambda^{2}$. The MRI obviously suffer from magnetic diffusion if this damping rate is comparable to the growth rate of the fastest growing mode $\lambda_{\mathrm{f}}$. In the ideal conducting limit $\lambda_{\mathrm{f}}$ has a growth rate of $\approx \Omega$. In the presence of resistivity, however, $\lambda_{\mathrm{f}}$ becomes larger and the associated growth rate is reduced (Jin 1996). Ignoring for a moment this resistivity dependence of $\lambda_{\mathrm{f}}$ and taking the ideal plasma values one expects a strong influence of magnetic diffusion for diffusivities larger than $\eta \approx \Omega \lambda_{\mathrm{f}}^{2} / 4 \pi^{2}$. Based on this simplified argument one can define a corresponding critical magnetic Reynolds number which can be shown to be given by $R e_{\mathrm{m} \text {,crit }} \approx 3 \beta / 2$. If $R e_{\mathrm{m}}$ is of the order of $R e_{\mathrm{m}, \mathrm{crit}}$, the MRI will be severely affected. In more drastic cases where $R e_{\mathrm{m}} \ll R e_{\mathrm{m} \text {,crit }}$ one might expect a complete quenching of the instability because every potentially unstable mode in the box no longer grows. The above derivation gives some useful estimates for homogeneous systems. In a stratified disk the situation is more complex. Because $\lambda_{\mathrm{f}} \propto \beta^{-1 / 2}$ and $\beta$ is a function of $z$ (recall that in our model $\beta$ initially varies from $\beta=100$ at the disk midplane to $\beta \approx 1.9$ at $z= \pm 2$ ), the critical value of diffusivity also depends on height. Under the assumption $\eta=$ const. this means that the $\lambda_{\mathrm{f}}$-mode is more damped at the disk midplane than at the vertical boundaries because $\lambda_{\mathrm{f}}$ increases with $z$. As a consequence, stratified systems are relatively less affected by magnetic diffusion than non-stratified systems.

We now turn to the presentation of the numerical results. Note first that the background field $B_{z} \propto \sin (2 \pi x)$ is free to decay completely by magnetic diffusion because it is not driven by external currents in contrast to simulations adopting a constant field or a field configuration with an initially non-vanishing mean $\langle\boldsymbol{B}(t=0)\rangle \neq 0$. In cases of high resistivity the background field may be diffused away so rapidly that it may have severe implications for on the linear growth of the instability. This effect is indeed observed as the present decline in the time evolution of magnetic energy from the sturt, presented in Fig. 5. No such a decline occurs in the ideal case (S1). Except for model S5 $\left(R e_{\mathrm{m}}=200\right)$ this initial decline lasts only a few orbits and stops at $t \approx 3$ when the exponential growth phase begins. The magnetic energy level reached at the end increases with increasing $R e_{\mathrm{m}}$ as expected from the concept of resistive damping as a competing effect to field amplification by the MRI. Note in this context that the energy value of the less diffusive case $\mathrm{S} 7\left(R e_{\mathrm{m}}=12000\right)$ lies below that of $\mathrm{S} 6\left(R e_{\mathrm{m}}=2000\right)$ in Fig. (5) which means no contradiction because of the higher initial $\beta$ in S7.

The high resistivity case S5 shows a rather interesting behavior. Here, diffusion is so strong that magnetic field amplification is never attained. The magnetic energy first decreases by $\approx 8$ orders of magnitude reaching a minimum at $t \approx 5$. It then continuously grows and settles in a plateau with $\approx 30 \%$ of its initial value between $t \approx 35$ and $t \approx 60$. At still later times $(t \gtrsim 60)$ the magnetic energy increases further and finally reaches approximately its initial level. We believe that the extreme initial drop in magnetic energy is caused by the rapid diffusion of the sinusoidal background field. This diffusion of the background field is associated with a decrease of the wavelength of the fastest growth below the grid scale. Magnetic energy at this stage can thus be generated only by unstable modes with small wavenumber which have, however, much lower growth rates.

The quantity $M R S R$ defined by Eq. (16) is found to decrease with decreasing $R e_{\mathrm{m}}$ in accordance with the magnetic energy level. A very low value of $M R S R=0.07$ is obtained for S5 which emphasizes the effect of resistivity on that important quantity. The dependence of $M R S R$ on $R e_{\mathrm{m}}$ can be understood in terms of different dissipation scales of the fluctuating field components. Namely, dissipation of $\boldsymbol{B}^{\prime}$ occurs on increasingly larger spatial scales than dissipation of $\boldsymbol{v}^{\prime}$ if the value of $R e_{\mathrm{m}}$ is decreased. As a likely consequence, the average correlation between fluctuating magnetic field components is reduced whereas velocity correlations are affected less by the resistive MRI still acting as driver for turbulent motions. This effect attracts attention with the smaller parameter $M R S R$, as observed. We also find outward angular momentum 


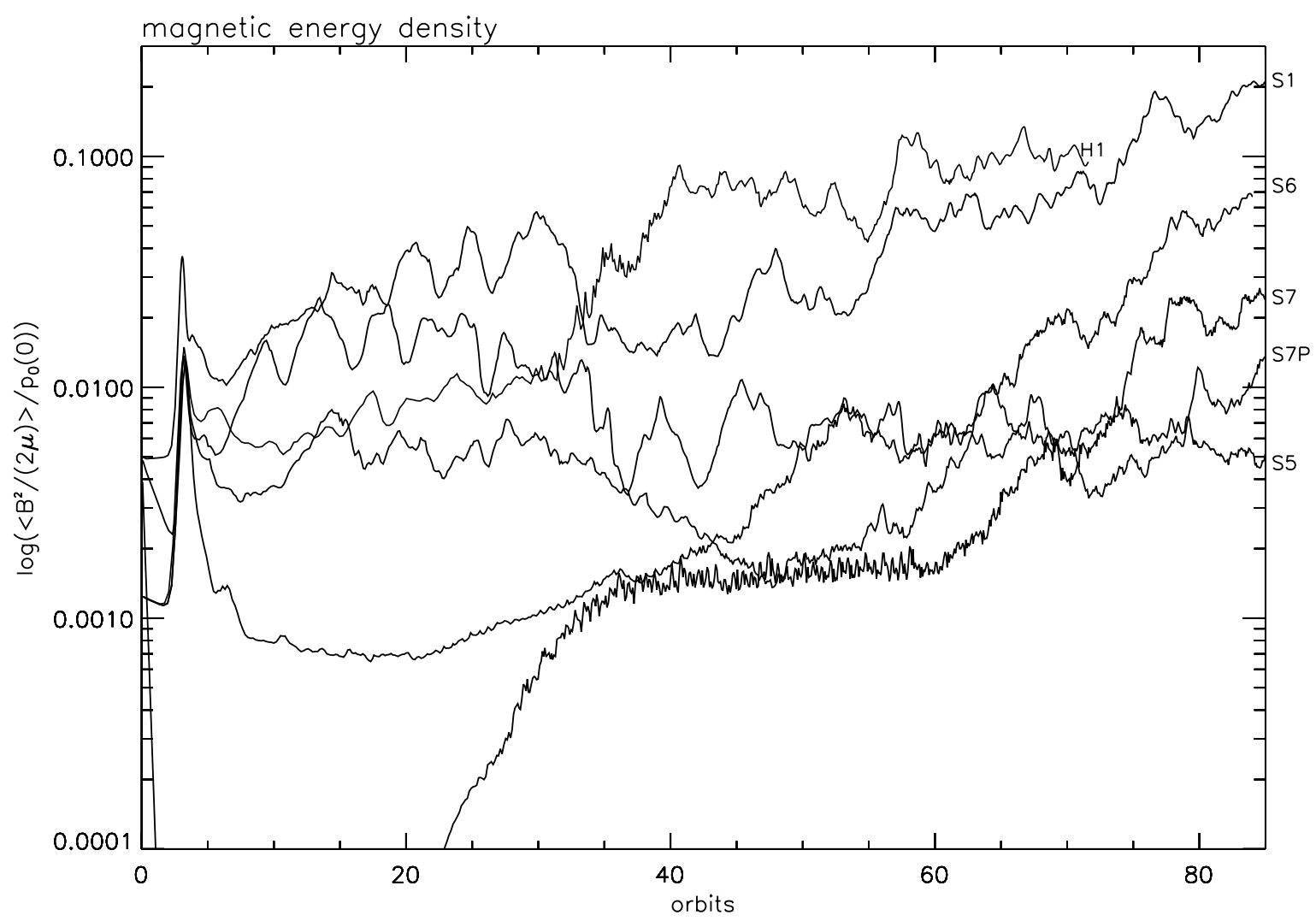

Fig. 5. Time evolution of the magnetic energy for different models. The thin line corresponds to the non-stratified model H1.

transport in all cases, albeit with less efficiency compared to the non-resistive models. The Shakura-Sunyaev parameter turns out to depend on the level of resistivity with a clear trend to smaller rates for higher resistivity. However, even for the most diffusive case S5, $\alpha_{\mathrm{SS}}$ has a magnitude roughly $1 / 3$ of that of an ideal plasma. In contrast to the ideal case, however, Reynolds stress provides a significant or even dominant contribution to $\alpha_{\text {SS }}$ consistent with the decline of $M R S R$. Note that Reynolds stress exceeds Maxwell stress by a factor of $\approx 14$ in $\mathrm{S} 5$.

The mean fields $\left\langle B_{y}\right\rangle^{\mathrm{N}}$ and $\left\langle B_{y}\right\rangle^{\mathrm{S}}$ are shown as a function of time in Fig. (6) for model S6. As in the non-resistive case, $\left\langle B_{y}\right\rangle^{\mathrm{N}}$ (and also $\left\langle B_{y}\right\rangle^{\mathrm{S}}$ ) shows cyclic behavior with an amplitude of the order of $B_{0}$ but its temporal signature is different from the ideal case. In particular, we find a constant phase between both mean field parts of $180^{\circ}$ i.e. the azimuthal magnetic field possesses a definite parity with respect to the diskplane. The existence of such a fixed phase relation is a further example of the influence of resistive damping. For decreasing $\eta$ the phase relationship between $\left\langle B_{y}\right\rangle^{\mathrm{N}}$ and $\left\langle B_{y}\right\rangle^{\mathrm{S}}$ becomes more and more complex and time-dependent, as has been found for nonresistive disks. The corresponding dynamo $\alpha$-parameter for both disk hemispheres is presented in Fig. 7. Analysis of the data yield $\left(\alpha^{* \mathrm{~N}}, \alpha^{* \mathrm{~S}}\right) \approx(-0.0030,0.0015)$ which is somewhat surprising, comparable in absolute value to models S0 and S1. We note here that at later evolutionary times there exists a mean magnetic field even for the high resistivity case $\mathrm{S} 5$. However, its amplitude $\left(\approx 0.1 B_{0}\right)$ is almost one order of magnitude smaller compared to S6.
The dynamo $\alpha$-effect in this case is rather small and can be considered consistent with zero within our numerical approach.

What is the effect of stratification and how does the vertical boundary condition influence the results? To answer these questions we have performed additional runs which serve as comparisons. $\mathrm{H} 1$ and $\mathrm{H} 2$ are homogeneous box simulations with periodic vertical boundary condition and with $\beta=400$ assuming either an ideal conducting plasma $(\mathrm{H} 2)$ or a resistive plasma characterized by $R e_{\mathrm{m}}=12000(\mathrm{H} 1)$. Another run, S7P, is identical to S7 except for the choice of the vertical boundary condition which is chosen periodic in S7P. Clearly, periodic vertical boundary conditions are inappropriate for a stratified disk. But it can help to gain some insight on the sensitivity of the results to condition (12). We find that all quantities listed in Table 1 are sensitive to our specific choice of boundary condition. In the periodic box model S7P, $M R S R$ is larger by $\approx 40 \%$ and $\alpha_{\mathrm{SS}}$ by $\approx 45 \%$ than in $\mathrm{S} 7$. Also, the $\alpha$-parameter is larger in $\mathrm{S} 7 \mathrm{P}$ and shows a higher degree of symmetry between the northern and southern disk hemisphere. The asymmetry in the $\alpha$-parameter obtained in almost all cases thus cannot be excluded with final certainty from being an artifact of the boundary condition (12). For validation, a better suited boundary condition for the problem under consideration would be desirable. We will keep this in mind for future modeling.

Non-stratified box simulations in the non-linear regime of the resistive MRI have been performed by FHS. FHS claimed that the MRI is unable to drive turbulence and to 


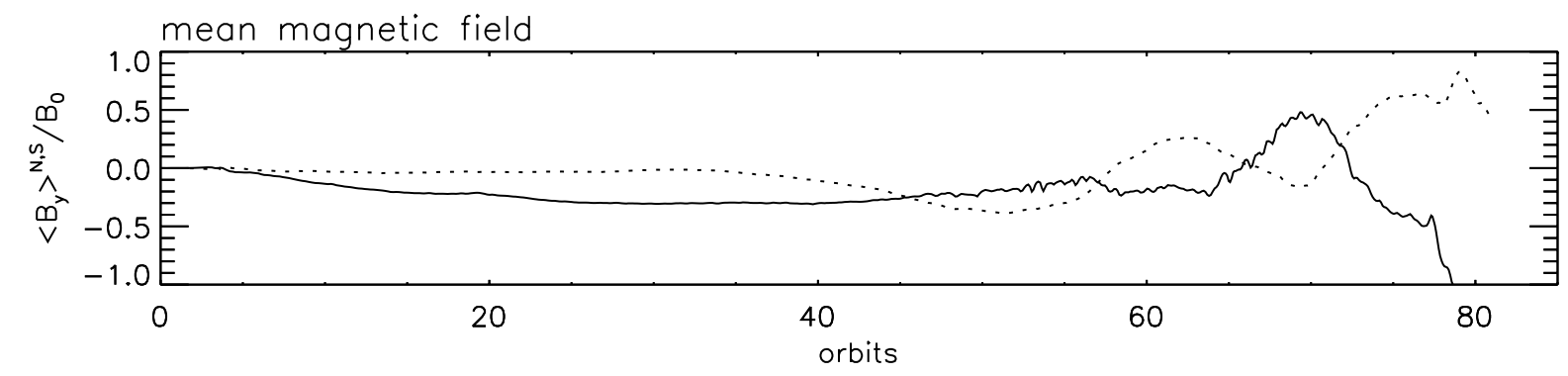

Fig. 6. Time history of $\left\langle B_{y}\right\rangle^{\mathrm{N}}$ (solid line) and $\left\langle B_{y}\right\rangle^{\mathrm{S}}$ (dotted line) for $\mathrm{S} 6\left(R e_{\mathrm{m}}=2000\right)$.
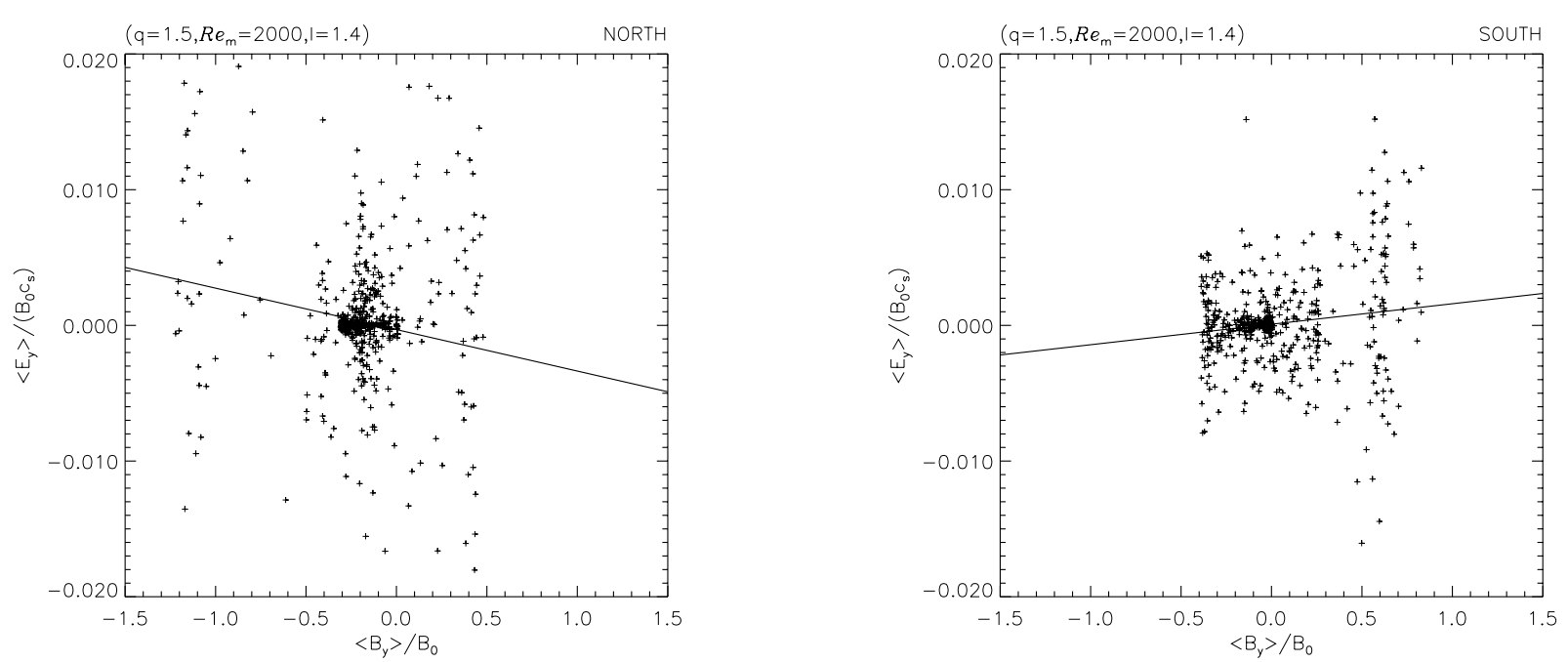

Fig. 7. $\alpha$-effect: correlation between the mean EMF and mean azimuthal magnetic field in the northern (left panel) and southern (right panel) hemisphere for model S6 $\left(R e_{\mathrm{m}}=2000\right)$.

sustain angular momentum transport against diffusion for magnetic Reynolds numbers $R e_{\mathrm{m}} \lesssim 10^{4}$. This finding is in gross contrast to the results we obtained for the stratified models. We find a significant angular momentum transport rate even for $R e_{\mathrm{m}}=200$. A possible explanation for this difference lies in the presence of stratification. Although, because of the $\lambda_{\mathrm{f}}$-dependence on height, stratified disks are indeed less affected by diffusion, it is unlikely that this effect can account for almost two orders of magnitude in the critical $R e_{\mathrm{m}}$. To shed more light on this problem, model $\mathrm{H} 1$ has been constructed to be most comparable to the vertical field net-flux simulation of FHS having $R e_{\mathrm{m}}=13000$. Despite different definitions of $R e_{\mathrm{m}}$ the magnetic diffusivities between this FHS model and H1 differ by less than 5 per cent. Again we do not find the drastic quenching visible in the FHS model (see Fig. 5 and compare $\mathrm{H} 1$ and $\mathrm{H} 2$ in Table 1). This obvious discrepancy constitutes a severe problem for which we cannot give a plausible explanation at the moment.

\section{Summary}

By means of numerical MHD simulations, we have studied the non-linear evolution of the MRI for different shear rates of the background rotational flow and in the presence of finite resistivity. The results were compared with those of a non-resistive Keplerian disk characterized by the appearance of self-sustaining, anisotropic turbulence, a positive rate of angular momentum transport mediated mainly by Maxwell stress, and the generation of a timedependent mean magnetic field in an azimuthal direction accompanied by dynamo $\alpha$-action where the $\alpha$-parameter is negative (positive) in the northern disk hemisphere.

Our survey of simulations at different shear rates give clear indication for a vanishing dynamo $\alpha$-effect for $q \lesssim 0.6$. At the same time, however, the angular momentum transport rate $\left(\alpha_{\mathrm{SS}}\right)$ does not vanish, expressing the possibility of accretion without involving a large-scale dynamo. Even for the lowest considered $q$-case $(q=0.6) \alpha_{\mathrm{SS}}$ reaches roughly $30 \%$ of the Keplerian value. In general, $\alpha_{\mathrm{SS}}$ is found to be a monotonically decreasing function of $q$. As $q$ decreases the contribution of the Reynolds stress to $\alpha_{\mathrm{SS}}$ becomes increasingly important and even dominates Maxwell stress in disks with low $q$. In those systems the turbulent flow is no longer magnetically dominated.

Finite resistivity does have an impact on the non-linear evolution of the MRI with consequences on dynamo processes and transport properties. The competing effect of resistive damping on unstable modes of the MRI leads to lower magnetic energy levels. Furthermore, the rate of angular momentum transport is reduced by diffusion. Both $\alpha_{\mathrm{SS}}$ and $M R S R$ decline with decreasing $R e_{\mathrm{m}}$. We observe 
dynamo $\alpha$-action in all resistive models except the case with $R e_{\mathrm{m}}=200$. For this high-resistivity run no field amplification takes place. Total suppression of the MRI thus, occurs at magnetic Reynolds numbers of the order of several hundred. This is in contradiction to results from homogeneous box simulations by FHS quoting a critical magnetic Reynolds number of $\approx 10^{4}$ for complete quenching. We concluded that this discrepancy in the critical magnetic Reynolds number cannot be explained by the presence of disk stratification alone, although on theoretical grounds stratified fluids with $\eta=$ const. are less affected by magnetic diffusion than non-stratified systems. In the cool, low states of dwarf novae one just expects magnetic Reynolds numbers in the regime $R e_{\mathrm{m}} \lesssim 10^{4}$ (Gammie $\&$ Menou 1998). In the light of our results magnetic diffusion seems to represent no severe restriction on the operation of the MRI in those systems. Also, in resistive planetary disks the MRI might work quit well as a source of turbulent motion and even as a magnetic field amplification mechanism. However, there are other restricting factors like ambipolar diffusion which lead to additional quenching effects (Blaes \& Balbus 1994; MacLow et al. 1995).

Finally, we don't want to conceal that our results are found to be sensitive to the choice of vertical boundary condition. In particular, forcing the magnetic field perpendicular at the $z$-boundaries as done in this study produces somewhat artificial currents at those locations which have a non-negligible influence on the general evolution with implications for mean parameters like $\alpha_{\mathrm{SS}}$. In this respect, the obtained results are not expected to constitute the final answer but rather give some estimates on the real behavior under various physical conditions. They should therefore be taken with some reservation until new results based upon more accurate boundary conditions are available.

Acknowledgements. This research was partially supported by the DFG under grant UL 57/30-1. The computations were performed on workstations at the Astrophysikalisches Institut Potsdam, Germany. We thank the referee for useful comments on the topic.

\section{References}

Abramowicz, M., Brandenburg, A., \& Lasota, J-P. 1996, MNRAS, 281, L21

Balbus, S. A., \& Hawley, J. F. 1991, ApJ, 376, 214

Balbus, S. A., \& Hawley, J. F. 1992, ApJ, 400, 610

Blaes, O. M., \& Balbus, S. A. 1994, ApJ, 421, 163

Brandenburg, A., Nordlund, A., Stein, R. F., \& Torkelsson, U. 1995, ApJ, 446, 741

Brandenburg, A., Nordlund, A., Stein, R. F., \& Torkelsson, U. 1996, ApJ, 458, L45

Brandenburg, A. 1998, in Theory of Black Hole Accretion Disks, ed. M. A. Abramowicz, G. Björnsson, \& J. E. Pringle, CUP, 61

Chandrasekhar, S. 1960, Proc. Natl. Acad. Sci., 46, 253

Fleming, T. P., Stone, J. M., \& Hawley, J. F. 2000, ApJ, 530, 464

Gammie, F., \& Menou, K. 1998, ApJ, 492, L75

Goodman, J., \& Xu, G. 1994, ApJ, 432, 213

Hawley, J. F., Gammie, F., \& Balbus, S. A. 1995, ApJ, 440, 742

Hawley, J. F., Gammie, F., \& Balbus, S. A. 1996, ApJ, 464, 690

Hawley, J. F., \& Stone, J. M. 1998, ApJ, 501, 758

Jin, L. 1996, ApJ, 457, 798

Krause, F., \& Rädler, K.-H. 1980, in Mean Field Magnetohydrodynamics and Dynamo Theory (Pergamon Press)

MacLow, M.-M., Norman, M. L., Konigl, A., \& Wardle, M. 1995, ApJ, 442, 726

Pouquet, A., \& Patterson, G. S. 1978, J. Fluid Mech., 85, 305

Rüdiger, G., \& Pipin, V. 2001, A\&A, 362, 756

Shakura, N. J., \& Sunyaev, R. A. 1973, A\&A, 24, 337

Stone, J. M., Hawley, J. F., Gammie, C. F., \& Balbus, S. A. 1996, ApJ, 463, 656

Stone, J. M., \& Norman, M. L. 1992a, ApJS, 80, 753

Stone, J. M., \& Norman, M. L. 1992b, ApJS, 80, 791

Stone, J. M., \& Norman, M. L. 1994, ApJ, 433, 746

Stone, J. M., Gammie, C. F., Balbus, S. B., \& Hawley, J. F. 2000, in Protostars and Planets IV, ed. V. Manning, A. Boss, \& S. Russell (Tucson: Univ. Arizona Press), in press Velikhov, E. P. 1959, Soviet Phys.-JETP, 36, 995

Ziegler, U. 1998, Comp. Phys. Comm., 109, 111

Ziegler, U. 1999, Comp. Phys. Comm., 116, 65

Ziegler, U., \& Rüdiger, G. 2000, A\&A, 356, 1141 